\title{
Microdroplet dye laser enhancing effects in dye-highly scattering intralipid mixture
}

\author{
Shinji Tanosaki and Hiroshi Taniguchi ${ }^{\text {a) }}$ \\ Department of Electrical and Electronic Engineering, Faculty of Engineering, Iwate University, \\ 4-3-5 Ueda, Morioka 020, Japan \\ Kazuhiro Tsujita \\ Biophotonics Information Laboratories, 2-2-1 Matsuei, Yamagata 990, Japan \\ Humio Inaba \\ Tohoku Institute of Technology, 35-I Yagiyama Kasumi-Cho, Taihaku-Ku, Sendai 982, Japan, and \\ Biophotonics Information Laboratories, 2-2-1 Matsuei, Yamagata 990, Japan
}

(Received 25 March 1996; accepted for publication 22 May 1996)

The significant effect of fat emulsion intralipid-10\% mixing on lasing behaviors of liquid microdroplets was studied using rhodamine $6 \mathrm{G}$ dye. This highly scattering medium allows one to make the high-gain laser dye-soft scatterers microsystems that can provide well-defined lasing threshold and one-order or more magnitude enhancement of emission intensity with suitable (optimum) conditions of the mixing ratio, in comparison with original neat-dye lasing microdroplets. We also present and discuss the measured results of input-output intensities for different dye concentrations and dye-intralipid mixing ratios, and microscope images of spatial distribution of emission from both the microdroplets containing neat-dye and dye-intralipid mixture.

(C) 1996 American Institute of Physics. [S0003-6951(96)02232-2]

Recently, the results of a series of experiments on laser action of optically pumped rhodamine dye solutions in strongly scattering media consisting of $\mathrm{TiO}_{2}$ nanoparticles have been reported. ${ }^{1,4}$ They described that multiple light scattering in a highly scattering and diffusive medium can trap excited photons as well as emitted photons in a small spatial region so that the gain exceeds the loss. ${ }^{1-5}$ Moreover, it was mentioned that the high-gain laser dye scattering system produces a narrow linewidth emission without any additional reflections. ${ }^{4}$ For the case of a micron-sized particle medium, discrete stationary modes owing to its distributed feedback-like configuration could be expected to improve such high-gain dye laser performance. About a decade ago, the group of one of the authors has demonstrated experimentally, for the first time to our knowledge, this fact by using rhodamine 6G (RH6G)-methanol solution containing spread layers of $\mathrm{OH}$-gel particles of about $5 \mu \mathrm{m}$ diameter, which exhibited considerably narrowed linewidth together with sharp output-beam pattern.

On the other hand, some interesting papers have reported currently on lasing from dye-doped microdroplets providing high- $Q$ optical cavities. ${ }^{7-9}$ These lasing emissions occur at discrete wavelengths corresponding to morphologydependent resonances (MDR's) (or to the whispering-gallery mode) of the sphere. From the viewpoint that microdroplets should contain various particulates, lasing behaviors were recently studied in liquid droplets containing fluorescent dye solution mixed fluorescent sol, ${ }^{10}$ polystyrene spheres, ${ }^{11}$ and undoped latex-sol particles. ${ }^{12}$ Most recently we have first reported the RH6G microdroplet dye-laser action accompanying well-defined thresholds together with appreciably increased emission intensities (not amplified spontaneous emission) containing highly scattering particulates, ${ }^{13}$ in

\footnotetext{
${ }^{a}$ Electronic mail: tani@dragon.elc.iwate-u.ac.jp
}

which the poly(methyl methacrylate) (PMMA) solid nanoparticles were used, although the intralipid solution as the strongly scattering turbid medium to simulate light propagation in biological tissues has been used in optical imaging experiments, ${ }^{14,15}$ however, no lasing action of immersed or mixed materials in this useful medium has been reported yet.

This letter reports experimental studies of microdroplet dye lasing from almost no-lasing neat-dye droplets which have much higher or much lower dye concentration, by adding a suitably fat emulsion intralipid solution as highly scattering media; well-defined lasing thresholds are observed when certain amounts of intralipid solution are mixed to the neat dye-doped microdroplets. We evidently found that the intralipid turbid solution added to microdroplets in neat-dye lasing at a nearly optimum dye concentration is also remarkably effective for enhancement of peak intensity for laser emission. Moreover, such dye-intralipid mixtures, with soft scatterers in contrast to those previous solid-nanoparticles appear to have much closer similarity to biocolloids in biological tissues and cells.

The experimental arrangement is similar to those described previously. ${ }^{9,13}$ We employed a frequency-doubled, $Q$-switched Nd:YAG laser (Nd:YAG second harmonic, 532 $\mathrm{nm}$ ) with $\sim 10$-ns pulse duration, and then its output beam is focused onto dye-doped liquid microdroplets. The weakly focused pump-beam size $(\sim 450-\mu \mathrm{m}$ diam) was larger than the dye (RH6G)-doped microdroplet. Spectra of light emitted from the dye-doped microdroplet irradiated by the laser are collected at $90^{\circ}$ to the incident pump beam and are analyzed with an optical multichannel analyzer (OMA) mounted upon a microscope. Proper-sized microdroplets formed around a fine horizontally strung tungsten fiber ( $\sim 5 \mu \mathrm{m}$ diam), by a simple method that inserts the fiber and then pulls back the fiber in turn, into and then from, water and glycerol (WG) solution containing RH6G dye. These RH6G-doped solu- 


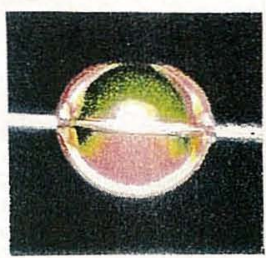

(a)

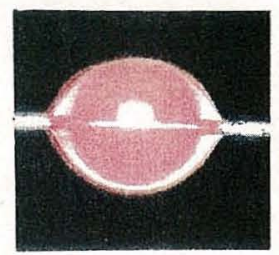

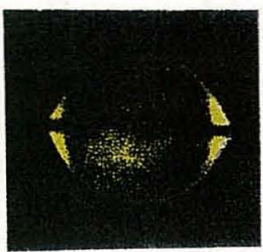

(b)

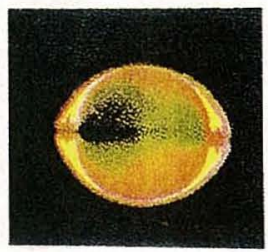

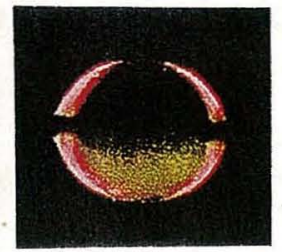

(c)

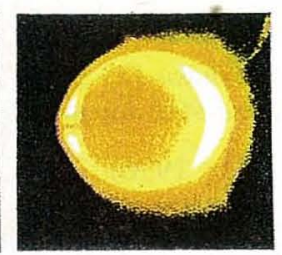

FIG. 1. Microscope image of suspended RH6G $\left(1 \times 10^{-3} \mathrm{M}\right)$-singlemicrodroplets compared with the cases of dye-intralipid mixture with $N_{c}$ $=\sim 18 \%$, (the lower figures) and neat-dye doped $\left(N_{c}=0 \%\right.$; the upper figures). (a) Droplet images obtained by white-light illumination, (b) in fluorescence (below threshold, $E_{p}=\sim 1.0-3.0$ in Fig. 4), and (c) in lasing (above threshold, $E_{p}=\sim 6.0-7.0$ in Fig. 4). Exposure of (c) is $\sim 1 / 20$ of (b).

tions are made from a WG mixture with a volume ratio of $\sim 10: 1$ for adequate viscosity.

Experimental studies were performed on the optical properties from a variety of concentrations of the RH6Gdoped spherical microdroplets (with diameter $D=\sim 100 \pm 5$ $\mu \mathrm{m})$ containing various amounts of intralipid solution (intralipid- $10 \%,{ }^{16}$ manufactured by Kabi Pharmacia AB, Uppsala, Sweden). We prepared various contents of the intralipid-10\% in the RH6G-doped WG-solution by changing volume ratios of the WG and the intralipid- $10 \%$ solutions.

First, we examined microscope images of spatial distribution of emission from the suspended single RH6Gmicrodroplets with a different quantity of intralipid-10\% scatterers, under the RH6G-dye concentration of $1 \times 10^{-3} \mathrm{M}$ as shown in Fig. 1; this concentration corresponds to nearly optimum so that it exhibits the lasing threshold even without intralipid scatterers (see Fig. 4). As explained in the figure caption, the lower and upper ones indicate the cases in the large amounts of the intralipid-10\% (volume content $N_{c}=$ $\sim 18 \%)$ and in the neat dye $\left(N_{c}=0 \%\right)$; (a) is the microdroplet image taken by white-light illumination with the microscope, and (b) and (c) are, respectively, the images in fluorescence and in lasing, by Nd:YAG second harmonic (532 $\mathrm{nm}$ ) pumping. Above the lasing threshold (c), the image of the emission pattern with the dye-intralipid mixture [the lower figure of (c)] shows a rather extended, uniform character accompanied with the rather brighter region around the center in contrast to that in the neat-dye microdroplet, where the rim appears brighter than the center [the upper figure of (c)]. In such a highly scattering medium, the mode structures owing to MDR's of spheres may disappear because the laser gain due to multiple light scattering inside the microsphere becomes dominant to exceed internal loss. Even in the cases of (a) and (b) in the lower pictures, one can observe the occurrence of such a multiple light scattering event compared with the upper ones for the neat-dye sample.

The emission intensity (from the RH6G-doped single

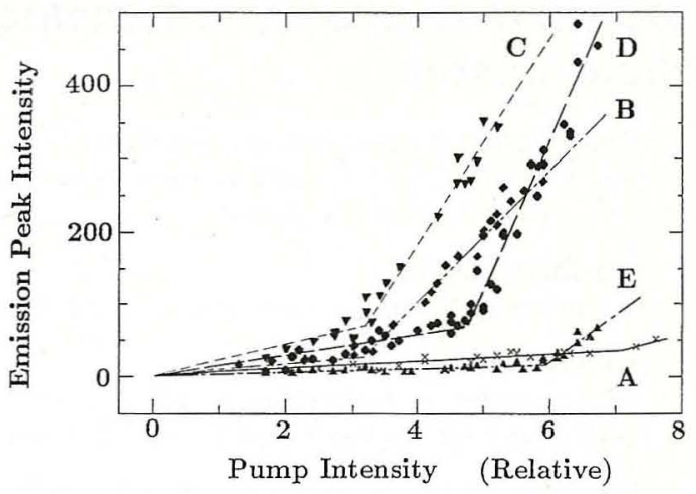

FIG. 2. Pump intensity $\left(E_{p}\right)$-dye microdroplet emission intensity characteristics containing $1 \times 10^{-5} \mathrm{M} \mathrm{RH6G}$, as a parameter of volume contents $\left(N_{c} \%\right)$ of the scattering intralipid- $10 \% . N_{c}=0 \%$ (plot A), $\sim 0.02 \%$ (B), $0.2 \%$ (C), $2 \%$ (D), and $4 \%(\mathrm{E})$.

droplet) at the peak wavelength is shown in Fig. 2 as a function of the relative pump-beam intensity $E_{p}$, as a parameter of volume contents $\left(N_{c} \%\right)$ of the scattering intralipid-10\%: $N_{c}=0 \%$ (plot A), $0.02 \%$ (B), $0.02 \%,(\mathrm{C}), 2 \%$ (D), and $4 \%$ (E), under a fixed much-lower dye concentration $\left(1 \times 10^{-5}\right.$ M RH6G). The RH6G neat droplet without the intralipid (plot A) exhibits a nearly linear slope in about $E_{p}<7$ of input-output intensities, whereas the microdroplets with intralipid reveal the drastic slope-change demonstrating the lasing thresholds at the bending points $\left[E_{p o}=\sim 3.2(\mathrm{~B}), 3.2\right.$ (C), 4.7 (D), and 6.0 (E)]. We remark that a symptom of lasing threshold is observable at the much higher pump intensity $\left(E_{p}>7\right)$ in such a much-lower neat dye concentration. No effects of highly scattering nanoparticles on these much-lower dye concentration solutions (even for bulk as well as for microdroplets) have been described, to the best of our knowledge.

For much-higher RH6G-dye concentration $\left(5 \times 10^{-3}\right.$ M), the analogous data to Fig. 2 are plotted in Fig. 3, compared with the neat dye $\left(N_{c}=0 \%\right.$ intralipid-10\%) WG solution (plot A); $N_{c}=\sim 2 \%$ (B), $18 \%$ (C), and 50\% (D). The neat droplet without the intralipid shows a tendency for an approximately linear dependence of input-output intensities, while the microdroplets containing the certain amounts of

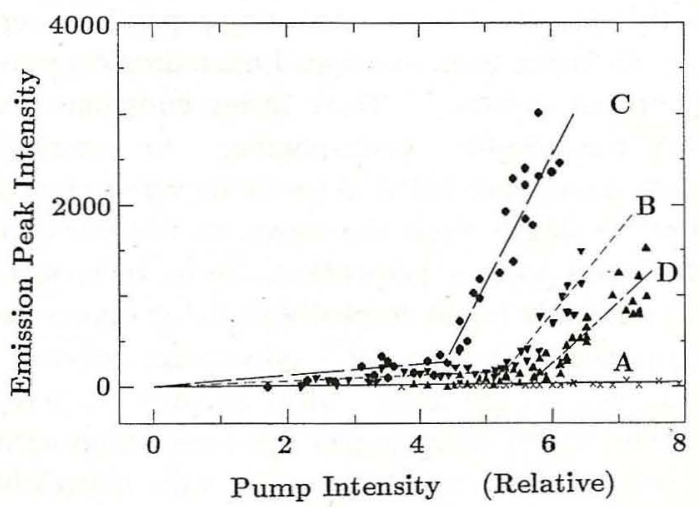

FIG. 3. Pump intensity $\left(E_{p}\right)$-dye microdroplet emission intensity characteristics containing $5 \times 10^{-3} \mathrm{M} \mathrm{RH} 6 \mathrm{G}$, as a parameter of volume contents $\left(N_{c} \%\right)$ of the scattering intralipid-10\%. $N_{c}=0 \%$ (plot A), $\sim 2 \%$ (B), $18 \%$ (C), and $50 \%$ (D). 


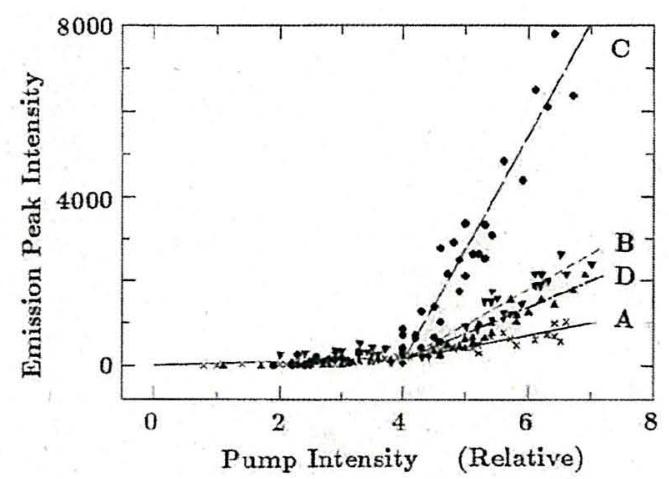

FIG. 4. Pump intensity $\left(E_{p}\right)$-dye microdroplet emission intensity characteristics containing $1 \times 10^{-3} \mathrm{M}$ RH6G, as a parameter of volume contents $\left(N_{c} \%\right)$ of the scattering intralipid-10\%. $N_{c}=0 \%$ (plot A), $\sim 2 \%$ (B), $18 \%$ (C), and $33 \%$ (D).

the intralipid (B-D) provide two distinct regions with bending points corresponding to the lasing thresholds $\left[E_{p o}=\right.$ - 5.2 (B), 4.4 (C), and 5.6 (D)]. No laser action could be obtained, as far as we know, in such a much-higher neat dye concentration, which resembles our previous results. ${ }^{13}$

Moreover, Fig. 4, with similar measurements to Figs. 2 and 3, comes from the case of the nearly optimum RH6Gdye concentration $\left(1 \times 10^{-3} \mathrm{M}\right)$ as is the case of Fig. 1, even in which the RH6G neat droplet $\left(N_{c}=0 \%\right.$ intralipid-10\%) without the intralipid (plot A) also exhibits the lasing threshold at the bending point $\left(E_{p o}=\sim 4.0\right)$, as well as the cases with the intralipid: $N_{c}=\sim 2 \%$ (B), $18 \%$ (C), and 33\% (D). In such a nearly optimum dye concentration, no distinctive differences are observed with respect to the lasing thresholds in this microdroplet; however, it is to be mentioned that the addition of the intralipid scatterers contribute remarkably to enhance the droplet laser emission (in Fig. 4, plot $\mathrm{C}$ produces $\sim 6$ times stronger than that in plot $\mathrm{A}$ at $E_{p}=\sim 6$ ).

We should also mention that even with the smaller droplets (e.g., $D \leqslant 60 \mu \mathrm{m}$, compared to the present $D=\sim 100$ $\mu \mathrm{m})$ within our OMA resolution, a tendency of the disappearance of the well-known periodic mode structures owing to MDR's of spherical cavities, when substituted intralipid$10 \%$ quantities are increased to the neat-dye droplets with normal MDR structures. This tendency also seems to be recognized by microscope observation of the spatial distribution of microdroplet dye emission as presented in Fig. 1. It is considered that in the cases of dye-intralipid mixture in much lower (Fig. 2) or much higher (Fig. 3) RH6G dye concentrations, optical gain contributed by multiple light scattering is much larger than that due to MDR's whereas in the case of optimum dye concentration (Fig. 4), the gain due to MDR's is first dominant in the neat microdroplet and then that produced by multiple light scattering becomes dominant in the dye-intralipid mixture. Generally, in neat droplets, laser intensity builds up through optical feedback owing to MDR's at the sphere-surrounding medium interface, while adding a scattering medium in the droplets should make it difficult to form MDR's, in which the laser intensity may build up through multiple light scattering in a small spatial region, just the same as those in bulk cells. ${ }^{1,5}$ Detailed measurements on mode structures are needed for further information of dye-microdroplet lasing behavior containing highly scattering nanoparticles. In the input-output characteristics shown in Figs. 2-4, the addition of the highly scattered intralipid$10 \%$ produces the different effects in the respective regions of the below and above lasing thresholds; there is not always a relation between laser threshold and slope efficiency. We infer from this fact that different contributions exist to photon buildup, in a multiple light scattering microsystem containing a highly scattering and diffusive medium unknown yet, between incoherently spontaneous (below thresholds) and coherently stimulated processes (above thresholds). We also remark that the RH-6G in WG solution exhibited the lower fluorescence quantum efficiency compared with that in organic solvents such as alcohol because of the probable aggregation of dye molecules. ${ }^{17}$ Nonetheless, the present dyeintralipid microsystem revealed the interesting lasing performance without additional deaggregating agent.

In conclusion, we have presented the first experimental results of interesting lasing properties of dye-doped spherical microdroplets substituted intralipid-10\% turbid solution as highly scattering media. It is our belief that the present method utilizing the highly scattering intralipid medium could be widely applied to a large number of dyes and similar lasing materials, and also the demonstrated results make this technique very attractive for a variety of practical applications.

This research was partly supported by a 1995 Grant-inAid from the Ministry of Education, Science, Sports and Culture, Japan [Grant (C) 07650041].

'N. M. Lawandy, R. M. Balachandran, A. S. L. Gomes, and E. Sauvain, Nature (London) 368, 436 (1994)

${ }^{2}$ N. M. Lawandy and R. M. Balachandran, Nature (London) 373, 204 (1995).

${ }^{3}$ W. Zhang, N. Cue, and K. M. Yoo, Opt. Lett. 20, 961 (1995).

${ }^{4}$ R. M. Balachandran and N. M. Lawandy, Opt. Lett. 20, 1271 (1995).

${ }^{5}$ A. Z. Genack and J. M. Drake, Nature 368, 400 (1994)

${ }^{6} \mathrm{~N}$. Chang, T. Suzuki, and H. Inaba, Oyo-Buturi (Jpn. Soc. of Appl. Phys.) 54, 841 (1985) (in Japanese).

${ }^{7}$ A. Biswas, H. Latifi, R. L. Armstrong, and R. G. Pinnick, Opt. Lett. 14, 214 (1989)

${ }^{8}$ A. J. Campillo, J. D. Eversole, and H.-B. Lin, Phys. Rev. Lett. 67, 437 (1991).

${ }^{9}$ H. Taniguchi and S. Tanosaki, Jpn. J. Appl. Phys. 32, L1421 (1993).

${ }^{10}$ R. L. Armstrong, J.-G. Xie, T. E. Ruekgater, and R. G. Pinnick, Opt. Lett. 17, 943 (1992).

${ }^{11}$ H. B. Lin, A. L. Huston, J. D. Eversole, A. J. Campillo, and P. Chylek, Opt. Lett. 17, 970 (1992).

${ }^{12}$ R. L. Armstrong, J.-G. Xie, T. E. Ruekgauer, J. Gu, and R. G. Pinnick Opt. Lett. 18, 119 (1993).

${ }^{13}$ H. Taniguchi, M. Nishiya, S. Tanosaki, and H. Inaba, Opt. Lett. 21, 263 (1996).

${ }^{14}$ K. P. Chan, M. Yamada, and H. Inaba, Electron. Lett. 30, 1753 (1994).

${ }^{15}$ K. P. Chan, M. Yamada, B. Devaraj, and H. Inaba, Opt. Lett. 20, 492 (1995).

${ }^{16}$ H. J. van Staveren, C. J. M. Moes, J. van Marle, S. A. Prahl, and M. J. C. van Gemert, Appl. Opt. 30, 4507 (1991). The constituents of $1000 \mathrm{~mL}$ of intralipid- $10 \%$, according to the manufacturer, are $100 \mathrm{~g}$ of soybean oil, $12 \mathrm{~g}$ of lecithin, $22.5 \mathrm{~g}$ of glycerol, and $861 \mathrm{~g}$ of water. Particle sizes of the constituents are dispersed in the nanometer range of $\sim 10-500 \mathrm{~nm}$ diam.

${ }^{17}$ K. H. Drexhage, Aggregation of Dye Molecules in Dye Lasers, edited by F. P. Schafer (Springer, Berlin, 1973), Chap. 4.5.4. 